\title{
Distinct biomarker signatures in HIV acute infection associate with viral dynamics and reservoir size
}

Jeffrey E. Teigler, ${ }^{1,2}$ Louise Leyre, ${ }^{3}$ Nicolas Chomont, ${ }^{3}$ Bonnie Slike, ${ }^{1,2}$ Ningbo Jian,,${ }^{1,2}$ Michael A. Eller, ${ }^{1,2}$ Nittaya Phanuphak, ${ }^{4}$ Eugène Kroon, ${ }^{4}$ Suteeraporn Pinyakorn, ${ }^{1,2}$ Leigh Anne Eller, ${ }^{1,2}$ Merlin L. Robb, ${ }^{1,2}$ Jintanat Ananworanich, ${ }^{1,2}$ Nelson L. Michael, ${ }^{1}$ Hendrik Streeck, ${ }^{1,2,5}$ Shelly J. Krebs, ${ }^{1,2}$ and RV254/RV217 study groups ${ }^{6}$

'US Military HIV Research Program, Walter Reed Army Institute of Research, Silver Spring Maryland, USA ${ }^{2}$ Henry M. Jackson Foundation, Bethesda, Maryland, USA. ${ }^{3} \mathrm{CRCHUM}$ and Department of Microbiology, Infectiology and Immunology, Université de Montréal, Montreal, Canada. ${ }^{4}$ SEARCH, The Thai Red Cross AIDS Research Centre, Bangkok, Thailand. ${ }^{5}$ Institute for HIV Research, University Hospital, University of Duisburg-Essen, Germany. ${ }^{6}$ The RV254/RV217 study group is detailed in the Supplemental Acknowledgments.

Estimating the size of the viral reservoir is critical for HIV cure strategies. Biomarkers in peripheral circulation may give insights into the establishment of the viral reservoir in compartments not easily accessible. We therefore measured systemic levels of 84 soluble biomarkers belonging to a broad array of immune pathways in acute HIV infection in both antiretroviral therapy-naive (ART-naive) individuals as well as individuals who began ART upon early detection of HIV infection. These biomarkers were measured longitudinally during acute and chronic infection and their relationship to viral reservoir establishment and persistence was assessed. We observed several distinct biomarker pathways induced following HIV infection such as IFN- $\gamma$-signaled chemokines, proinflammatory markers, and TNF- $\alpha$-family members. Levels of several of these factors directly correlated with contemporaneous viral loads and/or frequency of peripheral blood mononuclear cells harboring HIV DNA during acute HIV infection. MCP-1, MIP-3ß, sTNFR-II, and IL-10 levels prior to ART associated with HIV DNA levels after 96 weeks of treatment, suggesting a link between early immune signaling events and the establishment and persistence of the viral reservoir during ART. Furthermore, they offer potentially novel tools for gaining insight into relative reservoir size in acutely infected individuals and the potential of associated risks of treatment interruption.

Authorship note: $\mathrm{HS}$ and SJK contributed equally to this work.

Conflict of interest: JA has participated in advisory meetings for ViiV Healthcare and Merck.

Submitted: October 31, 2017

Accepted: April 12, 2018

Published: May 17, 2018

\section{Reference information:}

JCI Insight. 2018;3(10):e98420.

https://doi.org/10.1172/jici.

insight.98420.

\section{Introduction}

During the first few days of acute HIV-1 infection, viral replication begins at the site of infection and rapidly disseminates to many compartments of the body including lymphatic tissues, gut foci, and the central nervous system (CNS) (1). The spread of HIV can be augmented by the early onset of immune activation resulting from a variety of factors including innate sensing of viral gene products, danger signals from cell death, microbial translocation through a compromised gut epithelial barrier, and others $(2,3)$. Importantly, this acute time period is a decisive one in which reservoir establishment occurs. Once the viral reservoir is established it provides a source of persistent virus that cannot be eradicated by administration of antiretroviral therapy (ART) alone (1, 4-7). Despite the success of ART in reducing viral load to immeasurable levels in peripheral blood, HIV persists in quiescent cells that generate relatively minor, if any, viral antigens sensed by antiviral CD $8^{+}$ $\mathrm{T}$ cells, and are in privileged immunological sites where perfusion of ART can be substantially lower (8-16). While it is extremely difficult to directly assess these sites of HIV reservoir establishment, localized events in the tissue such as macrophage activation can lead to the production and release of inflammatory biomarkers detected in the periphery. Assessment of these systemic biomarkers could therefore allow insight into the processes of reservoir establishment and could yield important insights into interventions for HIV cure.

Acute HIV infection, like many other viral infections, results in a rapid and broad induction of many antiviral and proinflammatory biomarkers $(17,18)$. This cytokine storm was first described as induced within the first 1 to 2 weeks following detectable viremia. Biomarkers described within these studies that 
associated with early subtype B HIV infection included IFN- $\alpha$, IL-15, IL-6, IL-18, RANTES, IP-10, and TNF- $\alpha$ (19-22). It was first hypothesized that these markers represented a bulk immune activation state induced by massive T cell activation during acute HIV infection (23). Subsequent studies have suggested that biomarker expression in early HIV infection is not a single wave but rather follows an ordered pattern with certain markers such as IFN- $\alpha$ and IL-15 arising early but others such as TNF- $\alpha$ arising later in infection, representative of distinct immunological pathways $(17,24)$. These biomarker pathways are elicited during a crucial time period in acute HIV infection where many clinical parameters such as viral reservoir seeding occur. Therefore, it is critical to understand the kinetics with which different biomarker pathways are elicited during acute HIV infection and their potential linkage to viral reservoir seeding and persistence.

Despite continuous administration of ART and resultant suppression of HIV viremia, overall rates of non-AIDS mortalities and morbidities such as cardiovascular disease and neurological degeneration remain higher in treated HIV-infected individuals than in the general population (25-36). This observation coincides with low-level continuous elevation of many proinflammatory biomarkers despite effective ART, similar to those that occur late in life in HIV-uninfected individuals (37-42). While the cause of these increased inflammatory markers remains unclear and complex, several factors have been suggested, such as side effects of ART administration, immune reconstitution, ongoing microbial translocation from damaged mucosal sites, or low-level viral replication (43-53). In particular, several of these elevated markers are also induced during acute HIV infection and remain elevated throughout untreated chronic infection $(54,55)$. Knowing the decisive importance of events during acute infection, exploring a possible link between soluble biomarkers present in early acute infection and their relationship to viral reservoir seeding could therefore lead to an increased understanding of the processes of reservoir establishment and maintenance in treated HIV-infected individuals.

To assess the biomarker pathways induced during HIV infection and their linkage with HIV viral loads and reservoir establishment, we assessed systemic levels of 84 soluble biomarkers longitudinally in individuals during acute HIV infection that either remained ART-naive or received ART. These biomarkers were measured during viral upslope and peak viral load within acute infection as well as at 24 weeks and 96 weeks after infection. We observed significant associations of biomarkers with contemporaneous viral load and viral reservoir size in acute infection prior to ART administration. In particular, we also identified several biomarkers whose levels at treatment initiation in acute infection associated with levels of viral reservoir following 96 weeks of treatment. Together, these results indicate the utility of peripheral biomarker pathway signals to potentially gain insight into reservoir establishment during HIV acute infection. Furthermore, they yield potentially novel tools to indirectly assess viral reservoir seeding during acute HIV infection and to gauge the potential success of interventions in cure-related studies after treatment interruption.

\section{Results}

Biomarker pathway kinetics in acute HIV infection. In order to identify biomarkers induced following HIV infection as well as differences in pathway-induction kinetics, we began by measuring systemic levels of 84 biomarkers, including cytokines, chemokines, and soluble cytokine receptors, using Luminex multiplex assays in individuals beginning in early acute HIV infection. Ten Thai individuals from the HIV ECHO (RV217) acute infection cohort, who were treatment naive throughout the study duration (Supplemental Table 1 and Supplemental Figure 1; supplemental material available online with this article; https://doi.org/10.1172/ jci.insight.98420DS1) (56) were assessed for plasma biomarker levels at time points corresponding to the following stages: preinfection (day 0), early acute (days 1-4 after RNA-positive Aptima test [RNA ${ }^{+}$), peak acute (days 10-16 after $\mathrm{RNA}^{+}$), early chronic (days 240-267 after RNA ${ }^{+}$), and late chronic (days 647-782 after $\mathrm{RNA}^{+}$) time points. Analysis of median biomarker concentrations longitudinally revealed several temporal patterns of expression, with some biomarkers such as MCP-2 and sCD40L only showing relative increases during peak viral load, while others were induced predominantly in the later stages of HIV infection, such as BCA-1 and MIP-3 $\alpha$ (Figure 1). Notably, these different patterns of temporal expression made visible through heatmap analysis (Figure 1) revealed that not just individual biomarkers, but rather entire pathways displayed differential induction kinetics. Indeed, a subset of proinflammatory biomarkers including the monocyte chemoattractant cytokines MCP-1 and MCP-2 were induced during acute infection but resolved to levels observed prior to infection by early or late chronic infection (Supplemental Figure 2A). A second set of biomarkers including the TNF- $\alpha$ receptors sTNFR-I and sTNFR-II were induced primarily 


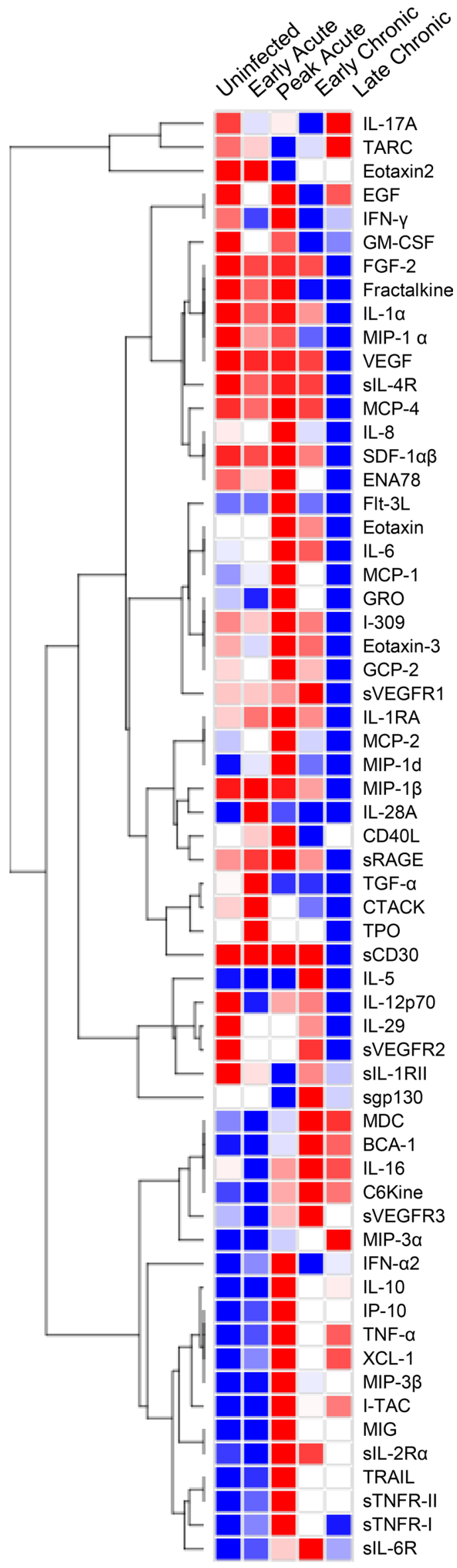

Figure 1. Biomarker pathways are induced in temporally distinct patterns following HIV infection. Eighty-four biomarkers were measured in plasma from HIV-infected individuals $(n=10)$ at uninfected (day 0), early acute (days 1-4), peak acute (days 10-16), early chronic (days 240-267), and late chronic (days 647-782) time points by Luminex assays. Unsupervised hierarchical clustering by Spearman's correlation of biomarkers differentially expressed between the 5 given time points. Colors indicate normalized row min-max shaded blue-red, respectively.

in acute infection but levels remained elevated in early chronic time points before returning to uninfected levels in late chronic infection. Levels of other biomarkers peaked in expression during peak viral load, and remained strongly elevated in chronic HIV infection, of which notable members were the IFN- $\gamma$-signaled chemokines MIG, IP-10, and I-TAC. Lastly, subsets of markers were substantially increased after the resolution of peak viremia. These markers included several markers of lymphocyte activation such as BCA-1, MIP-3 $\alpha$, and sIL-2R $\alpha$. Normalization of expression over time by $Z$ scores using HIV-uninfected time points as a reference further demonstrated the differential kinetics with which biomarkers are induced in HIV infection, with several being significantly expressed only during acute infection time points or peak viral load, but varying in levels of persistence in chronic infection, while others only arose significantly during chronic infection time points (Supplemental Figure 2B). Together, these data show that, rather than a uniform spike of expression during acute HIV infection, several various groups of biomarkers representing known biological pathways display differential induction kinetics during the course of HIV infection.

Biomarkers correlate with contemporaneous HIV viral loads. We next sought to identify biomarkers that were directly correlated with contemporaneous HIV viral loads during acute infection. Soluble biomarker levels were assessed in 40 Thai individuals at enrollment in the RV254 cohort at Fiebig Stage I ( $n=$ $10)$, II $(n=10)$, or III $(n=20)$ of acute HIV infection prior to the immediate administration of ART (Table 1) (57). In order to preserve the ability to compare between treated (RV254) and untreated (RV217) cohorts, individuals from the RV254 cohort were also Thai and mostly male (Supplemental Table 1). Levels of biomarkers were then assessed for correlations between one another and with contemporaneous HIV viral loads by Spearman correlogram analyses (Figure 2). Several biomarkers with known roles in inflammation and antiviral immune responses were observed to positively correlate with contemporaneous viral loads including IP-10, MIP-3 $\beta$, I-TAC, sTNFR-II, IL-10, MIG, sIL-6R, TNF- $\alpha$, MCP-1, and GRO (Figure 2 and Supplemental Table 2). Importantly, several of these biomarkers also correlated with one another, such as levels of IP-10, I-TAC, and MIP-3 $\beta$ or sTNFR-II and IL-10. Biomarkers that demonstrated the strongest correlations with viral loads and those that withstood Bonferroni's correction for multiple comparisons were IP-10 (rho $=0.753 ; P<0.0001$ ), MIP-3 $\beta$ (rho $=0.688, P<0.0001)$, I-TAC (rho $=0.63 ; P<0.0001)$, sTNFR-II (rho $=$ $0.581 ; P=0.0001)$, IL-10 (rho $=0.553 ; P=0.0003)$, and MIG (rho $=0.522 ; P=$ 0.0007 ) (Figure 3). While several of these identified markers were expected, as their presence in acute HIV infection had been previously described, we build on these observations by demonstrating that additional factors such as MIP-3 $\beta$, MIG, and I-TAC are expressed in acute HIV infection and correlate with contemporaneous HIV viral load. These data demonstrate that while several distinct biological pathways are activated during acute HIV infection, only a subset directly correlate with HIV viral load.

Continued elevation of HIV-associated biomarkers in individuals on ART. To determine whether HIV-associated biomarkers are similarly elevated in chronic infection when ART is initiated in acute infection, we compared their levels at early chronic (approximately 8 months after infection in RV217 or after ART initiation 
Table 1. Characteristics of RV254 individuals by infection stage upon diagnosis and enrollment

\begin{tabular}{cccc}
\hline RV254 & Fiebig I & Fiebig II & Fiebig III \\
Age & 10 & 10 & 20 \\
Male/Female & $27.5(18-46)$ & $28(25-34)$ & $29(21-46)$ \\
Enroll LogVL (copies/ml) & $8: 2$ & $8: 2$ & $19: 1$ \\
Enroll CD4 Abs (cells/ $\mu \mathrm{l})$ & $3.91(3.04-5.51)$ & $5.49(4.42-6.50)$ & $6.09(4.69-7.49)$ \\
\hline
\end{tabular}

Samples were provided and then individuals were immediately placed on treatment at this stage. Median values (range) are shown. VL, viral load.

in RV254 participants) and late chronic time points (approximately 2 years after infection in RV217 or after ART initiation in RV254 participants). Representative biomarkers from 3 different pathways linked to HIV viral load were downselected for analysis inclusive of TNF- $\alpha$ family members, lymphocyte activation markers, and IFN- $\gamma$-signaled chemokines. Despite early suppression of peripheral viremia in all individuals on therapy, levels of certain biomarkers from all 3 pathways remained elevated in ART recipients at early chronic infection (8 months, Figure 4). Interestingly, levels of several markers in ART recipients remained elevated even at late chronic time points ( 2 years), with levels of MIP-3 $\alpha$ and I-TAC significantly increased relative to HIV-uninfected individuals. This point is also demonstrated when the longitudinal levels of biomarkers after treatment initiation (RV254) is compared with the longitudinal levels of treatment-naive individuals (RV217) (Supplemental Figure 3), and these differences withstood the Kruskal-Wallis test for multiple comparisons. Indeed, several biomarkers such as IP-10, MIG, and I-TAC decrease during early chronic infection, similar to treatment-naive individuals, but their levels do not fully return to levels observed in uninfected individuals by 8 months after treatment for all 3 markers and at 2 years for I-TAC and MIP-3 $\alpha$ (Supplemental Figure 3). Therefore, biomarkers associated with HIV viral load remain elevated in chronic HIV infection relative to demographically matched uninfected individuals even in the presence of persistent ART when initiated in acute infection.

Soluble biomarker associations with viral reservoir dynamics. HIV persists in latently infected cells following treatment initiation, resulting in a reservoir of virus able to cause viral rebound upon treatment interruption. One measure of this reservoir is DNA copies of HIV in peripheral blood mononuclear cells (PBMCs). We therefore sought to determine whether the HIV-associated soluble biomarkers described above (Figure 2 and Supplemental Table 2) also associate with the size of the viral reservoir in acute infection prior to treatment initiation, and whether these biomarkers in acute infection aid prediction of the latent reservoir size after 96 weeks of treatment. Assessment of biomarker correlations with total HIV DNA in PBMCs revealed several associations with the size of the viral reservoir. Levels of IL-10, IP-10, MIG, sIL-2Ra, and sTNFR-II all significantly positively associated with levels of HIV DNA in acute infection, prior to treatment initiation (Figure 5). Similarly, we assessed Spearman correlations of HIV-associated biomarkers in acute infection with levels of HIV DNA after 96 weeks of treatment (Figure 6). Pretreatment levels of IL-10 (rho $=0.408 ; P=0.035$ ) and sTNFR-II (rho $=0.407 ; P=0.035$ ) and levels in acute infection significantly associated with the size of the reservoir after 96 weeks of treatment, with a trending association with MIP-3 $\beta$ (rho $=0.364 ; P=0.062$ ) (Figure 6). In addition to these markers, levels of HIV RNA at pre-ART correlated both with levels of HIV DNA present in PBMCs at week 0 (rho $=0.446 ; P=0.008$ ) as well as trended with HIV DNA levels following 96 weeks of therapy (rho $=0.374 ; P=0.054$ ) (Figures 5 and 6).

As levels of these biomarkers at treatment initiation were linked to contemporaneous HIV-1 RNA levels, we next wanted to determine if any of these markers could be predictive of viral reservoir size independent of contemporaneous viral load prior to treatment. Therefore, we performed linear regression of HIV-associated biomarkers and levels of HIV DNA in PBMCs at 96 weeks after treatment initiation while adjusting for HIV viral load during acute infection. After controlling for contemporaneous viral load, levels of MCP-1 at treatment initiation significantly and inversely associated with viral reservoir size after 96 weeks of treatment $(P=0.018)$. Since MCP-1 was the most significant factor after adjusting for viral load we took an additional step in building a multiple linear regression model by forward step-wise selection. We thus assessed the association of biomarkers and HIV-1 RNA in acute infection with HIV reservoir 96 weeks after treatment initiation. Using the additive method we observed the highest-performing model to include a negative association 


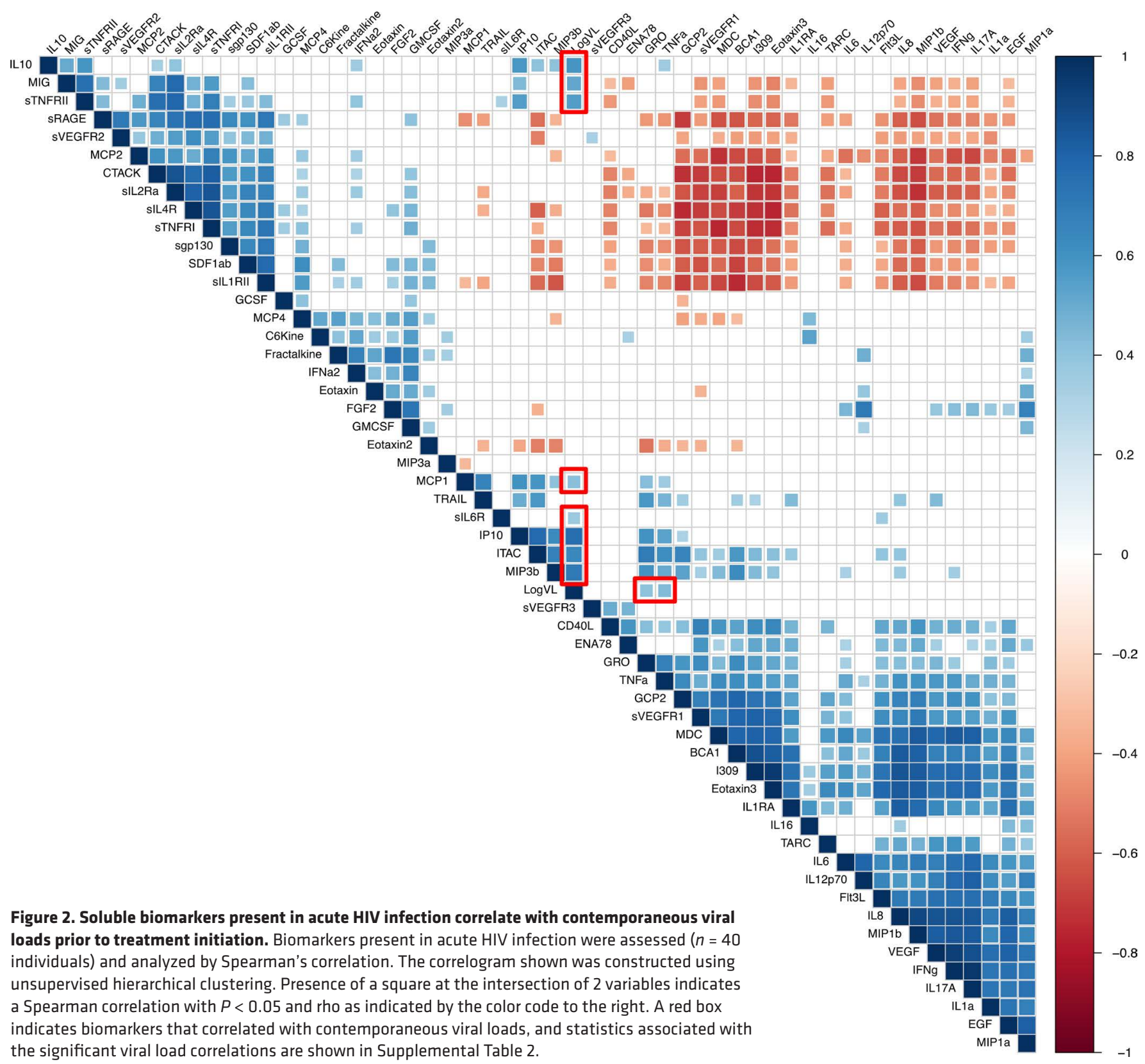

Figure 2. Soluble biomarkers present in acute HIV infection correlate with contemporaneous viral loads prior to treatment initiation. Biomarkers present in acute HIV infection were assessed $(n=40$ the significant viral load correlations are shown in Supplemental Table 2.

with MCP-1 and a positive association with sTNFR-II, MIP-3 $\beta$, and MIG when adjusted for $\log _{10}$ HIV-1 RNA (adjusted $R^{2}=0.3558, P=0.012$ ) (Table 2). These results show that biomarker levels in acute HIV infection, in addition to correlating with acute viral loads, can serve as useful markers to predict the levels of HIV DNA in PBMCs after treatment initiation, a surrogate for viral reservoir size.

\section{Discussion}

In these studies we identified potentially novel biomarkers associated with HIV viral load during acute HIV infection, and with the size of the viral reservoir prior to and following ART initiation. In the RV217 cohort of acutely HIV-infected individuals who did not receive ART, a pronounced and broad induction of several biomarker pathways was observed, with members of the proinflammatory, IFN- $\gamma$, and TNF- $\alpha$ pathways consistently being induced. Several of these pathways peaked coinciding with peak viral load, yet differentiated with respect to decay back to baseline levels when viral load reached the set point. Notably, while many biomarkers were induced following acute HIV infection, we only observed IP-10, I-TAC, MIP-3 sTNFR-II, IL-10, MIG, sIL-6R, TNF- $\alpha$, MCP-1, and GRO out of the 84 biomarkers assessed to be directly 

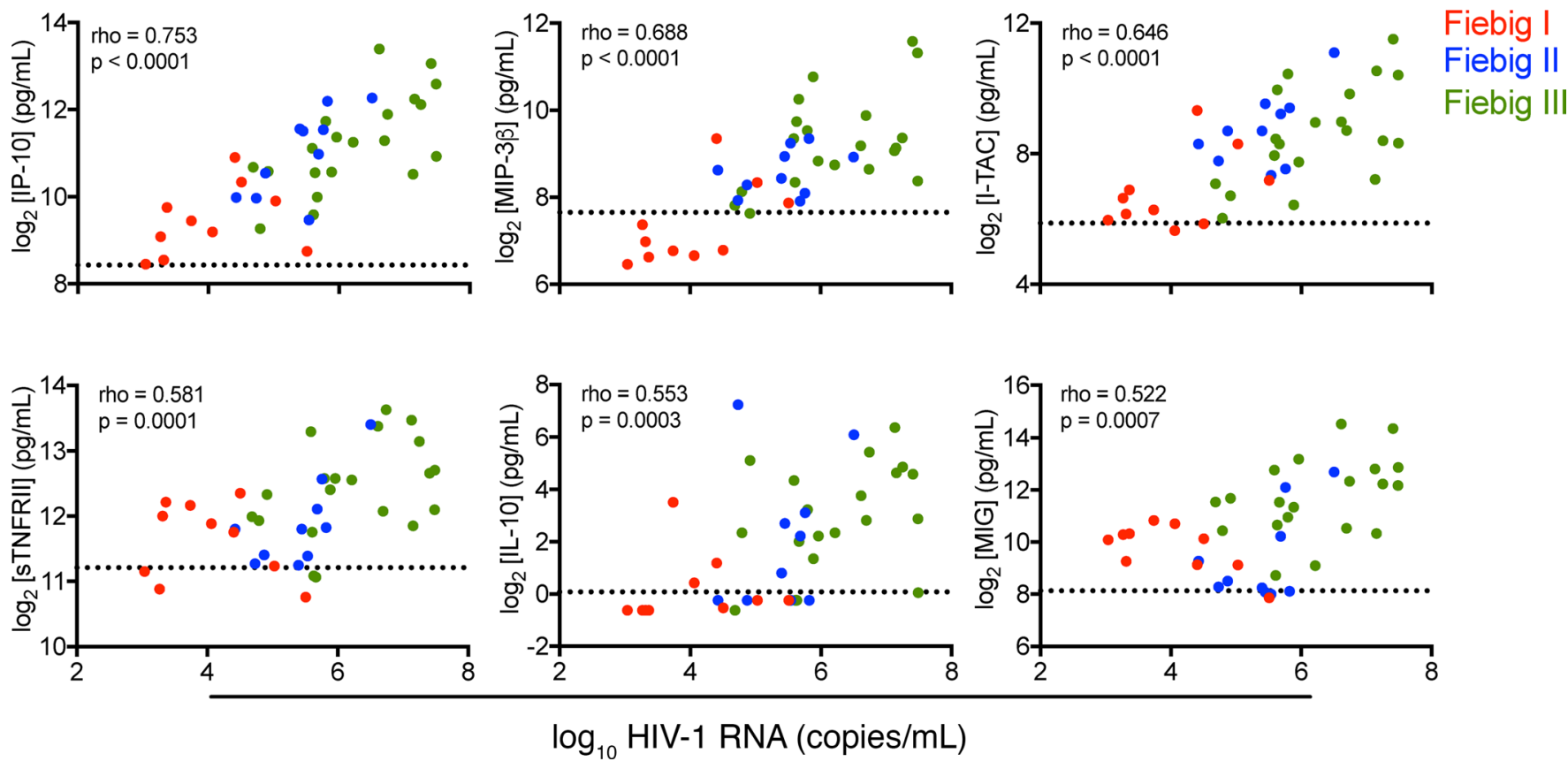

Figure 3. Soluble biomarkers present during acute HIV infection correlate with contemporaneous viral loads. Levels of biomarkers were assessed in individuals during acute HIV infection $(n=40)$ and correlated with contemporaneous HIV RNA copy levels. Markers were downselected based on those that remained significant following Bonferroni's correction for multiple comparisons. The color of the dots indicates the Fiebig stage of each individual when they entered the study in acute infection prior to treatment initiation. Dashed line represents mean biomarker concentration in HIV-uninfected individuals $(n=10)$. MIP-3b levels in Fiebig I were not significantly different than uninfected individuals, and a significant Spearman correlation with $P<0.05$ and rho $>0.5$ remained even after removing these individuals from the analysis.

linked with contemporaneous HIV viral loads. Interestingly, a subset of these markers subsequently correlated with levels of PBMC-associated HIV DNA prior to or after 96 weeks of treatment, suggesting that these markers may be indicative of signaling pathways induced at the site of viral replication or reservoir establishment during acute infection. Several biomarkers were observed that remained elevated relative to HIV-uninfected controls, in some cases up to 96 weeks after ART initiation, confirming and expanding on the observation that ART does not abrogate all aspects of immune activation in the setting of HIV infection, even when it is initiated in acute infection (54).

Previous studies have sought to characterize the timing and nature of immune activation immediately following HIV infection, and our studies presented here expand on these analyses to identify biomarker pathways associated with HIV dynamics. To the best of our knowledge, this is the broadest assessment of biomarkers carried out during acute HIV infection to date. We further expand on the previously identified pathways, identifying markers such as I-TAC, MIG, MCP-2, and GRO that are expressed during HIV acute infection. Furthermore, we identify a later class of biomarkers commonly associated with lymphocyte activation, such as BCA-1 and sIL-2R $\alpha$, which begin to rise in chronic infection. The majority of previous studies often focused on what was termed acute infection, but was rather either Fiebig IV/V or early chronic time periods, as these individuals were already past peak viral load (17, 19-22). In the cohorts that we presented here, individuals were captured either prior to (RV217) or within early acute infection Fiebig stage I, II, or III (RV254) and followed longitudinally. In addition, these cohorts are primarily subtype AE-infected individuals located in Thailand, as opposed to subtype B-infected individuals described in previous studies. These studies substantially expand on previous knowledge, identifying not only cardinal soluble inflammatory markers, but also full pathways of biomarkers induced with differential kinetics following acute HIV infection.

During acute HIV infection, immune activation results as a consequence of multiple events such as the translocation of and response to bacteria from a damaged gut, response to danger signals from bystander cell death, as well as sensing of viral antigens. For this reason, while a large number of biomarkers fluctuate during acute HIV infection, only a subset of these markers are reflective of viral 

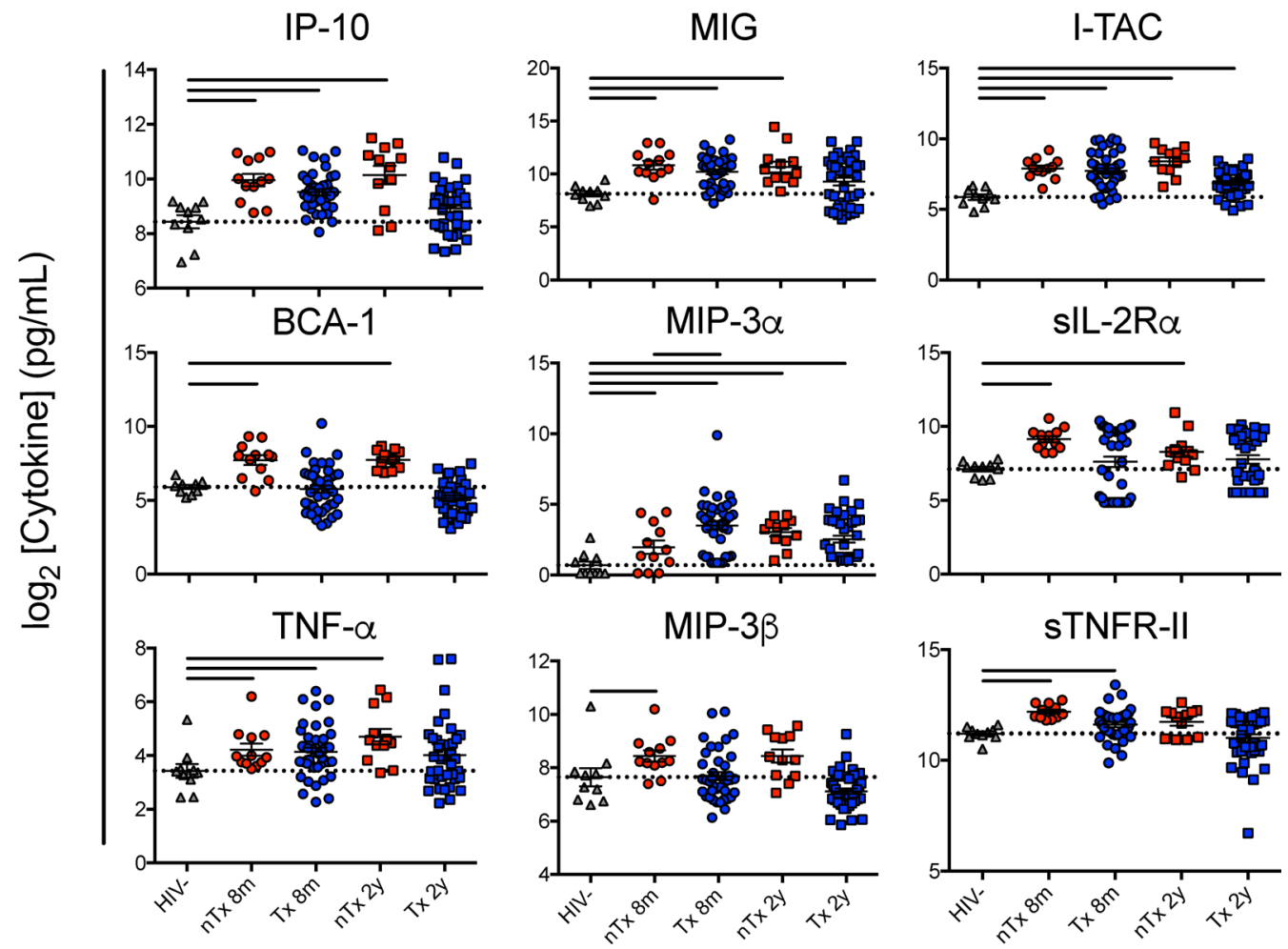

Figure 4. Levels of several soluble biomarkers that correlated with viral load persisted following treatment initiation. Systemic levels of biomarkers were assessed in HIV-uninfected individuals $(n=10)$, HIV-infected, treatment-naive $(n T x)$ individuals during early chronic (days $240-267$ [8m]) and late chronic (days 647-782 [2y]) $(n=10)$, or HIV-infected individuals with continuous treatment (Tx) initiated in acute HIV infection at time-period-matched sample stages $(n=40)$. Levels of selected biomarkers that correlated with acute HIV viral loads were then compared between all HIV-infected groups and uninfected individuals by Kruskal-Wallis test to account for multiple comparisons. Dashed line represents the median HIV-uninfected levels, and solid black lines indicate elevated levels relative to HIV-uninfected individuals with $P<0.05$. Data are presented as mean \pm SEM.

replication. In these studies we identified several biomarkers as significantly correlated with contemporaneous viral loads in acute HIV infection. While the function of these biomarkers is varied, they share the attribute that their production can be elicited from monocytes and macrophages by the sensing of IFN- $\alpha$ or IFN- $\gamma$. IFN- $\alpha$ and IFN- $\gamma$ can in turn be produced in response to viral antigens by several cells, including the sensing of HIV RNA by plasmacytoid DCs and T cell activation in response to novel antigens (58). The peripheral detection of IP-10, I-TAC, IL-10, MIP-3 $\beta$, and sTNFR-II may therefore represent spillover of biomarkers into the periphery in response to localized innate immune signals driven by HIV replication within the lymphoid compartments. It would be of interest for future studies to assess whether lymphoid sites are the main location at which these biomarkers are produced and whether their production represents a higher amount of viral replication within these sites.

The process by which the HIV reservoir is established and the cellular populations that comprise it has been extensively studied but remain incompletely understood. From the SIV model in nonhuman primates as well as efficacy studies and anecdotes from post-exposure prophylaxis (PEP), it is apparent that the reservoir of HIV infection is established extremely early (1). Indeed, the window over which ART can prevent permanent infection appears to be no longer than 2-3 days, after which the individual must undergo lifelong ART. The ability to understand in greater detail the processes and magnitude of reservoir establishment could enable the differentiation of subjects into HIV cure strategies. In these studies we identified several markers, including MCP-1, sTNFR-II, MIP-3 $\beta$, and MIG whose levels at ART initiation associate with levels of a surrogate marker for viral reservoir at 96 weeks after treatment initiation. It is intriguing that levels of MCP-1 (CCL2) stood out as the only factor observed to negatively associate with viral reservoir size. This could indicate that the induction of certain biological pathways, such as monocyte activation, early in HIV infection could help prevent reservoir establishment. Notably, several of these biomarkers are linked to the expression of IFN- $\alpha$, possibly via myeloid sensing of HIV replication 

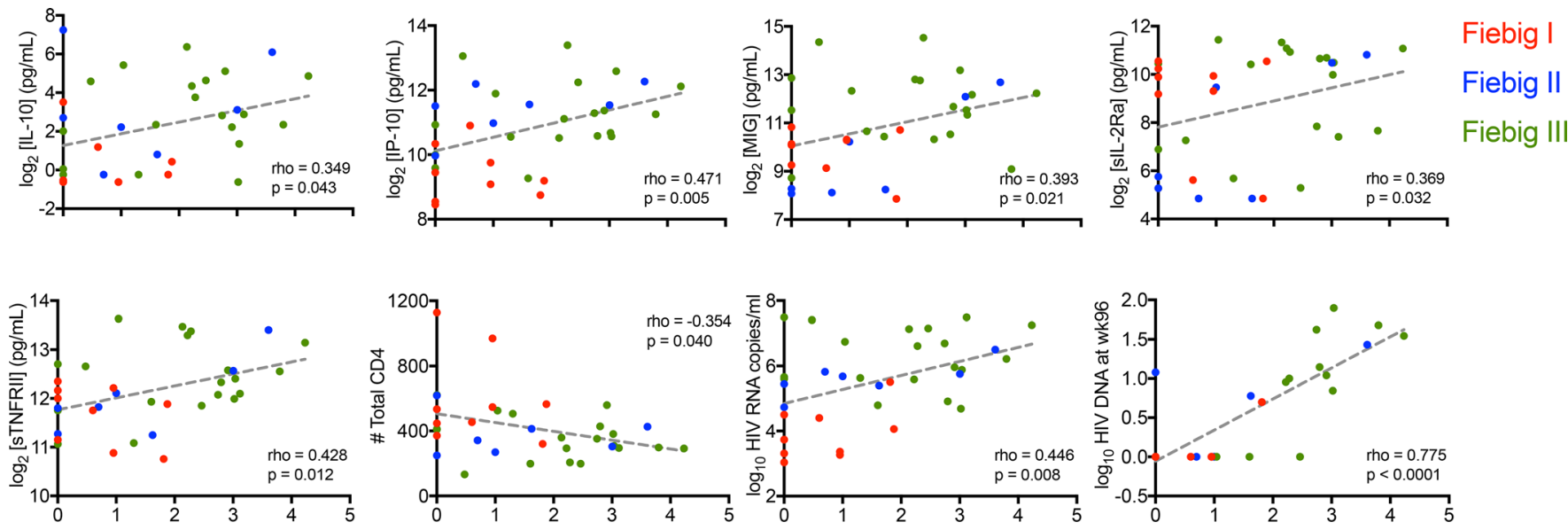

\section{$\log _{10}$ HIV DNA at wk0}

Figure 5. Soluble biomarker levels correlate with the size of the viral reservoir (total HIV DNA in peripheral PBMCs) prior to treatment initiation. Biomarkers were assessed in individuals diagnosed with HIV infection at Fiebig I $(n=10)$, Fiebig II $(n=10)$, or Fiebig III $(n=20)$. The color of the dots indicates the Fiebig stage of each individual when they entered the study. Levels of biomarkers were correlated with levels of total PBMC-associated HIV DNA levels at week 0 (wkO) prior to treatment initiation. The PBMC HIV DNA assay can detect a single copy of HIV DNA per PCR. The limit of detection depends on the number of cells examined and varies between samples. For simplicity, we represented all the samples with undetectable levels of HIV DNA on the $y$ axis as 0 . All comparisons shown are $P<0.05$ by Spearman's correlation.

products within lymphoid sites (58-60). This result stands in contrast to previous work that suggested no link between immune activation and levels of viral reservoir (61). However, these previous assessments were performed during chronic infection and not between acute biomarker levels and chronic reservoir levels. We also observed no link between biomarker levels in chronic infection and contemporaneous viral reservoir levels, confirming this prior work. Therefore, the levels of biomarkers in acute HIV infection could yield insight into reservoir establishment and greater inform HIV cure strategies.

The limitations of this study include the limited number of treatment-naive participants and treated individuals captured within each Fiebig stage. Increasing the number of individuals would have allowed for the evaluation of biomarker associations at each stage of early acute infection with viral reservoir size after treatment. In general, biomarker levels in Fiebig I tend to be lower than those at Fiebig II/III, due to lower viral load, and as such low signal-to-noise ratios make their utility a bit more nuanced for reservoir size prediction. In addition, although total levels of HIV DNA measured in PBMCs are a surrogate marker of viral reservoir size, it does not capture the actual size or complexity of reservoir establishment within lymphoid tissues. Additional studies need to be performed to further understand how the markers identified within this study relate to the mechanisms of immune activation at the site of establishment.

In this study, to our knowledge, we perform the broadest assessment of soluble biomarkers associated with acute HIV infection and viral reservoir size to date. We observed a marked induction of several temporally distinct biomarker pathways, which displayed differential resolution in chronic infection following ART administration. Importantly, we identified several biomarkers that were directly linked to HIV viral loads and yield insight into levels of viral reservoir seeding and the size of the latent reservoir. These results provide new tools to assess the reservoir prospectively in the context of intervention studies in order to assess reduction of the reservoir or viral rebound in treatment interruption settings. Validation of these biomarkers in intervention research seeking to achieve durable remission of viremia would permit a significant advance in our understanding of the nature of the reservoir and ability to select candidates for such interventions.

\section{Methods}

Experimental design. The goal of the study was to first identify pathways of biomarkers linked with HIV-1 viral load and reservoir size and to subsequently assess their relationship during chronic HIV infection in the presence of ART. To account for possible demographic effects, all selected individuals were Thai and were of similar age and sex (Table 1 and Supplemental Table 1). Sample number was chosen based 

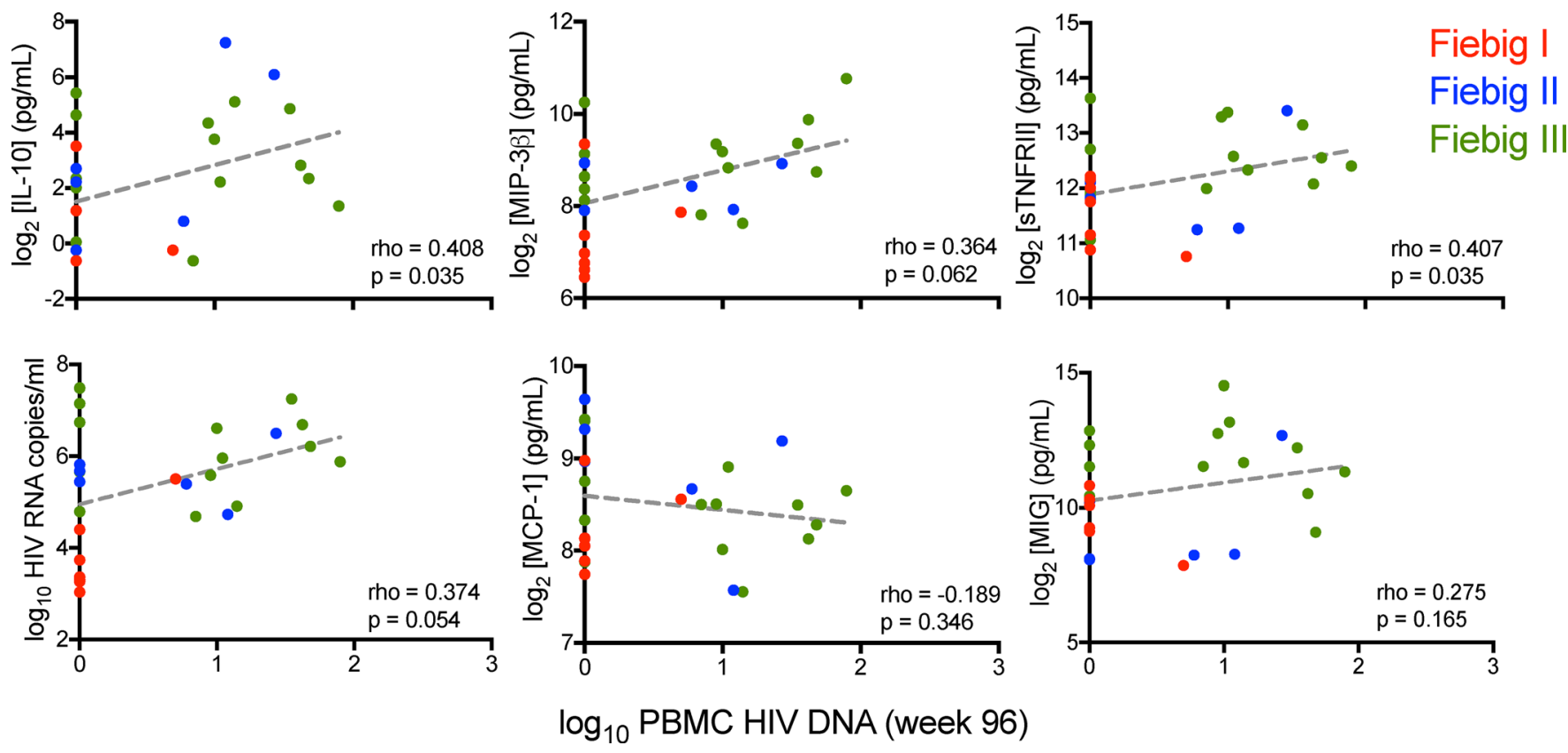

Figure 6. Soluble biomarker levels correlate with the size of the viral reservoir (total HIV DNA in PBMCs) 96 weeks following treatment initiation. Biomarkers were assessed in individuals diagnosed with HIV infection at Fiebig I $(n=10)$, Fiebig II $(n=10)$, or Fiebig III $(n=20)$. Linear regression of virus-associated biomarkers and chronic levels of HIV-1 PBMC DNA at 96 weeks after infection. The color of the dots indicates the Fiebig stage of each individual when they entered the study. Dashed gray line indicates best fit for simple linear regression model. Values indicate Spearman's rho and $P$ value for associated correlations with HIV DNA at 96 weeks after treatment initiation. The PBMC HIV DNA assay can detect a single copy of HIV DNA per PCR. The limit of detection depends on the number of cells examined and varies between samples. For simplicity, we represented all the samples with undetectable levels of HIV DNA on the $y$ axis as 0.

upon availability. Comparable sample time points were selected to incorporate viral upslope, peak, and 2 windows of chronic infection between the 2 studies with (RV254) and without (RV217) treatment.

Study participants. Plasma used was collected from peripheral blood processed in the presence of acidcitrate-dextrose (ACD) and subsequently cryopreserved. All subjects were enrolled in either the US Military HIV Research Program (MHRP) RV217 cohort or the Thai Red Cross AIDS Research Center RV254 cohort $(56,57)$. Cohort demographics are included in Table 1 and Supplemental Table 1. RV217 individuals were enrolled prior to HIV acquisition and tested with twice weekly finger pricks for positive HIV RNA using the sensitive Aptima test, as previously described (62). These individuals were then followed longitudinally in the absence of treatment, with intense sampling during acute infection (56). RV254 individuals were enrolled while in the Fiebig I, II, or III stage of acute HIV infection (Table 2), and immediately placed on treatment. Sampling was performed prior to treatment and longitudinally after treatment initiation (57).

Biomarker measurements. Cryopreserved ACD plasma was thawed on ice, clarified by centrifugation at $10,844 \mathrm{~g}$ for 10 minutes, and sterilized with $0.05 \%$ Tween 20 for 15 minutes at room temperature. Plasma was then measured for 84 biomarker levels using the Milliplex MAP Human Cytokine/Chemokine

Table 2. Soluble biomarker levels correlate with the size of the viral reservoir (total HIV DNA in PBMCs) 96 weeks following treatment initiation

\begin{tabular}{|c|c|c|c|c|}
\hline Marker & Estimate & Adj. Estimate & $P$ value & Adj. $P$ value \\
\hline MCP-1 & -0.603 & -0.675 & 0.0094 & 0.0064 \\
\hline sTNF-RII & 0.472 & 0.411 & 0.0377 & 0.077 \\
\hline MIP-3 $\beta$ & 0.330 & 0.24 & 0.0054 & 0.097 \\
\hline MIG & -0.111 & -0.12 & 0.242 & 0.207 \\
\hline
\end{tabular}

Biomarkers were assessed in individuals diagnosed with HIV infection at Fiebig I $(n=10)$, Fiebig II $(n=10)$, or Fiebig III ( $n=20)$. Multiple linear regression estimates and $P$ values for biomarkers found to yield best prediction of chronic viral reservoir size when controlled for contemporaneous HIV-1 RNA at treatment initiation. Values shown are for biomarker loading and significance in models unadjusted and adjusted for HIV-1 RNA levels. 
Magnetic Bead Panels I, II, III, and Milliplex MAP Human Soluble Cytokine Receptor Panel according to the manufacturer's instructions (EMD Millipore), as previously described (63). Samples were analyzed on a 5-parameter logistic curve with a standard acceptance range of $80 \%-120 \%$ on a BioPlex 200 running BioPlex Manager v6 (Bio-Rad).

Viral load and reservoir measurement. Plasma HIV RNA and total HIV DNA within PBMCs were measured as previously described $(56,64)$.

Statistics. GraphPad Prism (v7.0a) or R Studio (v1.0.44, R Consortium) was used to analyze data in a univariate fashion. For both univariate correlations and multivariate linear regression analyses, biomarker levels were $\log _{2}$ transformed prior to analysis in order to normalize distributions. Heatmaps were generated in Gene-E utilizing these $\log _{2}$-transformed values (Broad Institute) and correlograms were generated using the corrgram package in $\mathrm{R}(65) . Z$ scores were calculated by standard definition of value distance from group mean divided by individual biomarker's standard deviation in uninfected individuals. Significant differences between groups were determined by Mann-Whitney $U$ tests or Kruskal-Wallis test for multiple comparisons, and correlations were determined by Spearman's analyses. For all statistical tests performed, a 2-tailed $\alpha$ level was assumed, and $P<0.05$ was treated as significant unless otherwise noted. Due to cohort size and the exploratory nature of the study, Bonferroni corrections were employed in select analyses. For multiple linear regression analyses, the factor with the lowest $P$ value in simple linear regression was chosen. Factors were added to the model step-wise, with the model providing the highest adjusted $R^{2}$ employed as the basis for the subsequent model. Model construction was ended when addition of other variables did not improve adjusted $R^{2}$. Adjusted $R^{2}$ was employed to avoid model overfitting.

Study approval. All study participants gave written informed consent prior to inclusion in the study and approval was obtained from the Walter Reed Army Institute of Research (Silver Spring, MD), and the Chulalongkorn University and Phramongkutklao Hospital (Bangkok, Thailand) IRBs.

\section{Author contributions}

JET, HS, and SJK conceived and designed the research study. JET performed Luminex experimentation and data analysis with assistance from NJ, BS, and SP. LL and NC performed HIV reservoir measurements. JET and SJK wrote the manuscript. NP, EK, SP, LAE, MLR, and JA provided support to the clinical studies utilized to generate these data. MAE, JA, MLR, and NLM provided critical intellectual analysis and leadership to carry out these studies.

\section{Acknowledgments}

We would like to thank the participants involved in this study, as well as the study group members. See Supplemental Acknowledgments for RV254/SEARCH 010 Study Group details. This work was supported by cooperative agreements (W81XWH-07-2-0067 and W81XWH-11-2-0174) between The Henry M. Jackson Foundation for the Advancement of Military Medicine, Inc., and the US Department of Defense (DoD). JA is partially funded by NIH grant R01NS084911. Antiretroviral therapy was supported by the Thai Government Pharmaceutical Organization, Gilead, Merck, and ViiV Healthcare. The views expressed are those of the authors and should not be construed to represent the positions of the US Army or DoD.

Address correspondence to: Shelly J. Krebs, 503 Robert Grant Avenue; 1N29, Silver Spring, Maryland 20910, USA. Phone: 301.319.3961; Email: skrebs@hivresearch.org.

1. Whitney JB, et al. Rapid seeding of the viral reservoir prior to SIV viraemia in rhesus monkeys. Nature. 2014;512(7512):74-77.

2. Tabb B, et al. Reduced inflammation and lymphoid tissue immunopathology in rhesus macaques receiving anti-tumor necrosis factor treatment during primary simian immunodeficiency virus infection. J Infect Dis. 2013;207(6):880-892.

3. Stockmann M, et al. Mechanisms of epithelial barrier impairment in HIV infection. Ann N Y Acad Sci. 2000;915:293-303.

4. Joos B, et al. HIV rebounds from latently infected cells, rather than from continuing low-level replication. Proc Natl Acad Sci USA. 2008;105(43):16725-16730.

5. Wong JK, et al. Recovery of replication-competent HIV despite prolonged suppression of plasma viremia. Science. 1997;278(5341):1291-1295.

6. Finzi D, et al. Identification of a reservoir for HIV-1 in patients on highly active antiretroviral therapy. Science. 1997;278(5341):1295-1300.

7. Strain MC, et al. Effect of treatment, during primary infection, on establishment and clearance of cellular reservoirs of HIV-1 
J Infect Dis. 2005;191(9):1410-1418.

8. Haase AT. Population biology of HIV-1 infection: viral and CD4+ T cell demographics and dynamics in lymphatic tissues. Annu Rev Immunol. 1999;17:625-656.

9. Perreau M, et al. Follicular helper T cells serve as the major CD4 T cell compartment for HIV-1 infection, replication, and production. J Exp Med. 2013;210(1):143-156.

10. Yukl SA, et al. The distribution of HIV DNA and RNA in cell subsets differs in gut and blood of HIV-positive patients on ART: implications for viral persistence. J Infect Dis. 2013;208(8):1212-1220.

11. Pissani F, Streeck H. Emerging concepts on T follicular helper cell dynamics in HIV infection. Trends Immunol. 2014;35(6):278-286.

12. Quigley MF, Gonzalez VD, Granath A, Andersson J, Sandberg JK. CXCR5+ CCR7- CD8 T cells are early effector memory cells that infiltrate tonsil B cell follicles. Eur J Immunol. 2007;37(12):3352-3362.

13. Connick E, et al. CTL fail to accumulate at sites of HIV-1 replication in lymphoid tissue. J Immunol. 2007;178(11):6975-6983

14. Fletcher CV, et al. Persistent HIV-1 replication is associated with lower antiretroviral drug concentrations in lymphatic tissues. Proc Natl Acad Sci USA. 2014;111(6):2307-2312.

15. Chun TW, Engel D, Berrey MM, Shea T, Corey L, Fauci AS. Early establishment of a pool of latently infected, resting CD4(+) T cells during primary HIV-1 infection. Proc Natl Acad Sci USA. 1998;95(15):8869-8873.

16. Yukl SA, et al. Effect of raltegravir-containing intensification on HIV burden and T-cell activation in multiple gut sites of HIVpositive adults on suppressive antiretroviral therapy. AIDS. 2010;24(16):2451-2460.

17. Stacey AR, et al. Induction of a striking systemic cytokine cascade prior to peak viremia in acute human immunodeficiency virus type 1 infection, in contrast to more modest and delayed responses in acute hepatitis B and C virus infections. $J$ Virol. 2009;83(8):3719-3733.

18. Biglino A, et al. Serum cytokine profiles in acute primary HIV-1 infection and in infectious mononucleosis. Clin Immunol Immunopathol. 1996;78(1):61-69.

19. Birx DL, Redfield RR, Tencer K, Fowler A, Burke DS, Tosato G. Induction of interleukin-6 during human immunodeficiency virus infection. Blood. 1990;76(11):2303-2310.

20. Iketleng T, et al. Plasma cytokine levels in chronic asymptomatic HIV-1 subtype C infection as an indicator of disease progression in Botswana: a retrospective case control study. AIDS Res Hum Retroviruses. 2016;32(4):364-369.

21. Gay C, et al. Cross-sectional detection of acute HIV infection: timing of transmission, inflammation and antiretroviral therapy. PLoS ONE. 2011;6(5):e19617.

22. Norris PJ, Pappalardo BL, Custer B, Spotts G, Hecht FM, Busch MP. Elevations in IL-10, TNF-alpha, and IFN-gamma from the earliest point of HIV type 1 infection. AIDS Res Hum Retroviruses. 2006;22(8):757-762.

23. Graziosi C, et al. Kinetics of cytokine expression during primary human immunodeficiency virus type 1 infection. Proc Natl Acad Sci USA. 1996;93(9):4386-4391.

24. Kenway-Lynch CS, Das A, Pan D, Lackner AA, Pahar B. Dynamics of cytokine/chemokine responses in intestinal CD4 ${ }^{+}$and $\mathrm{CD}^{+} \mathrm{T}$ cells during acute simian immunodeficiency virus infection. J Virol. 2013;87(21):11916-11923.

25. d'Ettorre G, et al. What happens to cardiovascular system behind the undetectable level of HIV viremia? AIDS Res Ther. 2016;13:21.

26. Baker J, et al. High-density lipoprotein particles and markers of inflammation and thrombotic activity in patients with untreated HIV infection. J Infect Dis. 2010;201(2):285-292.

27. Kalayjian RC, et al. Pretreatment levels of soluble cellular receptors and interleukin- 6 are associated with HIV disease progression in subjects treated with highly active antiretroviral therapy. J Infect Dis. 2010;201(12):1796-1805.

28. Lederman MM, Funderburg NT, Sekaly RP, Klatt NR, Hunt PW. Residual immune dysregulation syndrome in treated HIV infection. Adv Immunol. 2013;119:51-83.

29. Valcour VG, Shikuma CM, Watters MR, Sacktor NC. Cognitive impairment in older HIV-1-seropositive individuals: prevalence and potential mechanisms. AIDS. 2004;18 Suppl 1:S79-S86.

30. Ellis RJ, et al. CD4 nadir is a predictor of HIV neurocognitive impairment in the era of combination antiretroviral therapy AIDS. 2011;25(14):1747-1751

31. Arpadi SM, Horlick M, Thornton J, Cuff PA, Wang J, Kotler DP. Bone mineral content is lower in prepubertal HIV-infected children. J Acquir Immune Defic Syndr. 2002;29(5):450-454.

32. Teichmann J, et al. Changes in calciotropic hormones and biochemical markers of bone metabolism in patients with human immunodeficiency virus infection. Metab Clin Exp. 2000;49(9):1134-1139.

33. Amorosa V, Tebas P. Bone disease and HIV infection. Clin Infect Dis. 2006;42(1):108-114.

34. Hsue PY, et al. Progression of atherosclerosis as assessed by carotid intima-media thickness in patients with HIV infection. Circulation. 2004;109(13):1603-1608.

35. Valcour V, Paul R. HIV infection and dementia in older adults. Clin Infect Dis. 2006;42(10):1449-1454

36. Desquilbet L, et al. HIV-1 infection is associated with an earlier occurrence of a phenotype related to frailty. J Gerontol A Biol Sci Med Sci. 2007;62(11):1279-1286.

37. Kamat A, et al. A plasma biomarker signature of immune activation in HIV patients on antiretroviral therapy. PLoS ONE. 2012;7(2):e30881.

38. Shaw AC, Joshi S, Greenwood H, Panda A, Lord JM. Aging of the innate immune system. Curr Opin Immunol. 2010;22(4):507-513.

39. Nixon DE, Landay AL. Biomarkers of immune dysfunction in HIV. Curr Opin HIV AIDS. 2010;5(6):498-503.

40. McKay HS, et al. Host factors associated with serologic inflammatory markers assessed using multiplex assays. Cytokine. 2016;85:71-79.

41. Neuhaus J, et al. Markers of inflammation, coagulation, and renal function are elevated in adults with HIV infection. $J$ Infect Dis. 2010;201(12):1788-1795.

42. Deeks SG, Tracy R, Douek DC. Systemic effects of inflammation on health during chronic HIV infection. Immunity. 2013;39(4):633-645

43. Giorgi JV, et al. Shorter survival in advanced human immunodeficiency virus type 1 infection is more closely associated with T lymphocyte activation than with plasma virus burden or virus chemokine coreceptor usage. J Infect Dis. 1999;179(4):859-870. 
44. Funderburg NT, et al. Increased tissue factor expression on circulating monocytes in chronic HIV infection: relationship to in vivo coagulation and immune activation. Blood. 2010;115(2):161-167.

45. Hunt PW, et al. Gut epithelial barrier dysfunction and innate immune activation predict mortality in treated HIV infection J Infect Dis. 2014;210(8):1228-1238.

46. Schuetz A, et al. Initiation of ART during early acute HIV infection preserves mucosal Th17 function and reverses HIV-related immune activation. PLoS Pathog. 2014;10(12):e1004543.

47. Havlir DV, et al. Predictors of residual viremia in HIV-infected patients successfully treated with efavirenz and lamivudine plus either tenofovir or stavudine. J Infect Dis. 2005;191(7):1164-1168.

48. Maldarelli F, et al. ART suppresses plasma HIV-1 RNA to a stable set point predicted by pretherapy viremia. PLoS Pathog. 2007;3(4):e46

49. Palmer S, et al. New real-time reverse transcriptase-initiated PCR assay with single-copy sensitivity for human immunodeficiency virus type 1 RNA in plasma. J Clin Microbiol. 2003;41(10):4531-4536.

50. Palmer S, et al. Low-level viremia persists for at least 7 years in patients on suppressive antiretroviral therapy. Proc Natl Acad Sci USA. 2008;105(10):3879-3884

51. Olesen R, et al. Innate immune activity correlates with CD4 T cell-associated HIV-1 DNA decline during latency-reversing treatment with panobinostat. J Virol. 2015;89(20):10176-10189.

52. Cockerham LR, et al. CD4 ${ }^{+}$and $\mathrm{CD} 8^{+} \mathrm{T}$ cell activation are associated with HIV DNA in resting CD4 ${ }^{+} \mathrm{T}$ cells. PLoS One. 2014;9(10):e110731.

53. Hatano $\mathrm{H}$, et al. Cell-based measures of viral persistence are associated with immune activation and programmed cell death protein 1 (PD-1)-expressing CD4+ T cells. J Infect Dis. 2013;208(1):50-56.

54. Sereti I, et al. Persistent, albeit reduced, chronic inflammation in persons starting antiretroviral therapy in acute HIV infection Clin Infect Dis. 2017;64(2):124-131.

55. Krebs SJ, Ananworanich J. Immune activation during acute HIV infection and the impact of early antiretroviral therapy. Curr Opin HIV AIDS. 2016;11(2):163-172.

56. Robb ML, et al. Prospective study of acute HIV-1 infection in adults in East Africa and Thailand. N Engl J Med 2016;374(22):2120-2130.

57. Ananworanich J, et al. HIV DNA set point is rapidly established in acute HIV infection and dramatically reduced by early ART. EBioMedicine. 2016;11:68-72.

58. Kader M, et al. Blocking TLR7- and TLR9-mediated IFN- $\alpha$ production by plasmacytoid dendritic cells does not diminish immune activation in early SIV infection. PLoS Pathog. 2013;9(7):e1003530.

59. Beignon AS, et al. Endocytosis of HIV-1 activates plasmacytoid dendritic cells via Toll-like receptor-viral RNA interactions J Clin Invest. 2005;115(11):3265-3275.

60. Mandl JN, et al. Divergent TLR7 and TLR9 signaling and type I interferon production distinguish pathogenic and nonpathogenic AIDS virus infections. Nat Med. 2008;14(10):1077-1087.

61. Gandhi RT, et al. Levels of HIV-1 persistence on antiretroviral therapy are not associated with markers of inflammation or activation. PLoS Pathog. 2017;13(4):e1006285.

62. Manak MM, et al. Identification of acute HIV-1 infection by Hologic Aptima HIV-1 RNA Qualitative assay. J Clin Microbiol. 2017;55(7):2064-2073.

63. Teigler JE, Phogat S, Franchini G, Hirsch VM, Michael NL, Barouch DH. The canarypox virus vector ALVAC induces distinct cytokine responses compared to the vaccinia virus-based vectors MVA and NYVAC in rhesus monkeys. $J$ Virol. 2014;88(3):1809-1814.

64. Vandergeeten C, et al. Cross-clade ultrasensitive PCR-based assays to measure HIV persistence in large-cohort studies. $J$ Virol. 2014;88(21):12385-12396.

65. Wright K. Package “corrgram”: plot a correlogram. R-project. https://CRAN.R-project.org/package=corrgram. Published May 7, 2017. Accessed April 19, 2018. 